\title{
Optimal timing for prediction of pathologic complete response to neoadjuvant chemoradiotherapy with diffusion-weighted MRI in patients with esophageal cancer
}

\author{
Alicia S. Borggreve ${ }^{1,2}$ (D) Sophie E. Heethuis ${ }^{1} \cdot$ Mick R. Boekhoff $^{1} \cdot$ Lucas Goense $^{1,2}$ • Peter S. N. van Rossum ${ }^{1}$. \\ Lodewijk A. A. Brosens ${ }^{3} \cdot$ Astrid L. H. M. W. van Lier ${ }^{1} \cdot$ Richard van Hillegersberg $^{2} \cdot$ Jan J. W. Lagendijk $^{1} \cdot$ Stella Mook $^{1}$ • \\ Jelle P. Ruurda ${ }^{2} \cdot$ Gert J. Meijer $^{1}$
}

Received: 27 May 2019 / Revised: 18 September 2019 / Accepted: 14 October 2019 / Published online: 10 December 2019

(C) The Author(s) 2019

\begin{abstract}
Objective This study was conducted in order to determine the optimal timing of diffusion-weighted magnetic resonance imaging (DW-MRI) for prediction of pathologic complete response (pCR) to neoadjuvant chemoradiotherapy (nCRT) for esophageal cancer.

Methods Patients with esophageal adenocarcinoma or squamous cell carcinoma who planned to undergo nCRT followed by surgery were enrolled in this prospective study. Patients underwent six DW-MRI scans: one baseline scan before the start of nCRT and weekly scans during 5 weeks of nCRT. Relative changes in mean apparent diffusion coefficient (ADC) values between the baseline scans and the scans during nCRT $(\triangle \mathrm{ADC}(\%))$ were compared between pathologic complete responders (pCR) and non-pCR (tumor regression grades 2-5). The discriminative ability of $\triangle \mathrm{ADC}(\%)$ was determined based on the $c$-statistic.

Results A total of 24 patients with 142 DW-MRI scans were included. pCR was observed in seven patients $(29 \%) . \Delta \mathrm{ADC}(\%)$ from baseline to week 2 was significantly higher in patients with pCR versus non-pCR (median [IQR], 36\% [30\%, 41\%] for pCR versus $16 \%[14 \%, 29 \%]$ for non-pCR, $p=0.004)$. The $\triangle \mathrm{ADC}(\%)$ of the second week in combination with histology resulted in the highest $c$-statistic for the prediction of pCR versus non-pCR (0.87). The $c$-statistic of this model increased to 0.97 after additional exclusion of patients with a small tumor volume $(<7 \mathrm{~mL}, n=3)$ and tumor histology of the resection specimen other than adenocarcinoma or squamous cell carcinoma $(n=1)$.

Conclusion The relative change in tumor ADC $(\triangle \mathrm{ADC}(\%))$ during the first 2 weeks of nCRT is the most predictive for pathologic complete response to nCRT in esophageal cancer patients.

Key Points

- DW-MRI during the second week of neoadjuvant chemoradiotherapy is most predictive for pathologic complete response in esophageal cancer.

- A model including $\triangle A D C_{\text {week } 2}$ was able to discriminate between pathologic complete responders and non-pathologic complete responders in $87 \%$.

- Improvements in future MRI studies for esophageal cancer may be obtained by incorporating motion management techniques.
\end{abstract}

Electronic supplementary material The online version of this article (https://doi.org/10.1007/s00330-019-06513-0) contains supplementary material, which is available to authorized users.

Alicia S. Borggreve

A.S.Borggreve@umcutrecht.nl

Gert J. Meijer

G.J.Meijer@umcutrecht.nl

1 Department of Radiation Oncology, University Medical Center

Utrecht, Utrecht University, Heidelberglaan 100, 3584, CX

Utrecht, The Netherlands
2 Department of Surgery, University Medical Center Utrecht, Utrecht University, Heidelberglaan 100, 3584, CX Utrecht, The Netherlands

3 Department of Pathology, University Medical Center Utrecht, Utrecht University, Heidelberglaan 100, 3584, CX Utrecht, The Netherlands 
Keywords Esophageal cancer $\cdot$ Chemoradiotherapy $\cdot$ Neoadjuvant treatment $\cdot$ Diffusion-weighted magnetic resonance imaging · Organ-sparing treatment

$\begin{array}{ll}\text { Abbreviations } \\ \text { ADC } & \text { Apparent diffusion coefficient } \\ \text { CTV } & \text { Clinical target volume } \\ \text { DW-MRI } & \text { Diffusion-weighted magnetic resonance imaging } \\ \text { GTV } & \text { Gross target volume } \\ \text { IQR } & \text { Interquartile range } \\ \text { MANEC } & \text { Mixed adenoneuroendocrine carcinoma } \\ \text { nCRT } & \text { Neoadjuvant chemoradiotherapy } \\ \text { NSA } & \text { Number of signal averages } \\ \text { pCR } & \text { Pathologic complete response } \\ \text { PTV } & \text { Planning target volume } \\ \text { ROC } & \text { Receiver-operating characteristic } \\ \text { SD } & \text { Standard deviation } \\ \text { TRG } & \text { Tumor regression grade } \\ \text { tT2W } & \text { Transverse anatomical T2-weighted } \\ \text { UICC } & \text { Union for International Cancer Control }\end{array}$

Introduction

Neoadjuvant chemoradiotherapy (nCRT) followed by esophagectomy is considered the standard of care for locally advanced, resectable esophageal cancer without distant metastases [1, 2]. Through tumor downsizing and downstaging, nCRT improves locoregional control and overall survival rates compared to surgery alone [2-4]. The degree of tumor regression in response to nCRT is directly related to long-term survival, with pathologic complete response $(\mathrm{pCR})$ resulting in the most favorable long-term prognosis $[4,5]$. A pCR, defined as the absence of viable tumor cells at the site of the primary tumor after nCRT, is observed in around 16-29\% of the patients after nCRT $[2,6,7]$. For these pathologic complete responders, it is questioned whether they benefit from a subsequent esophagectomy, which is associated with substantial morbidity and impaired quality of life [8-13]. In order to study the safety and feasibility of postponing or even omitting esophagectomy in these patients, accurate prediction of complete responders is essential.

Diffusion-weighted magnetic resonance imaging (DWMRI) is one of the modalities that is actively studied for its potential in treatment response assessment in multiple cancers, including esophageal cancer [14-20]. DW-MRI is an appealing imaging technique because it is a quantitative method, noninvasive, relatively fast, and without exposure to ionizing radiation [21]. It depends on the microscopic mobility of water and is highly influenced by tissue cellularity and tissue organization [21]. Treatment with chemoradiotherapy can result in the loss of cell membrane integrity and apoptosis, and this process can be detected as an increase in the mean tumor apparent diffusion coefficient (ADC) [22]. However, a subsequent decrease in tumor ADC values may occur by fibrosis, which may complicate interpretation and predictive ability for treatment response [23]. Previous studies have shown promising results for DW-MRI before nCRT, as well as during the first 2-3 weeks of nCRT in the prediction of pathologic response in esophageal cancer patients $[14,15$, 24-27]. To investigate and further optimize the predictive ability of DW-MRI during nCRT for response assessment in esophageal cancer, this study aimed at establishing the optimal timing of DW-MRI scanning during nCRT for the prediction of $\mathrm{pCR}$ in patients with esophageal squamous cell carcinoma and adenocarcinoma.

\section{Methods}

This single-center, prospective cohort study was approved by the institutional review board of the University Medical Center Utrecht (protocol ID 15-340). All participants provided written informed consent. The primary aim of the study was to research intrafraction tumor motion and regression in order to develop patient-specific adaptive radiotherapy using MRI [28, 29]. The current analysis on the optimal timing for response prediction was a prespecified secondary aim of this prospective study; hence, not all patients who were eligible for inclusion in the prospective study were analyzed in the current analysis.

\section{Study population}

Consecutive patients with histologically confirmed squamous cell carcinoma or adenocarcinoma of the esophagus or gastroesophageal junction who were scheduled to undergo nCRT followed by esophagectomy between December 2015 and April 2018 were eligible for inclusion in the current analysis. Exclusion criteria for enrollment in the study included age $<18$ years, previous treatment with thoracic surgery or thoracic radiotherapy, and contraindications for MRI. Exclusion criteria for the current analysis included unexpected distant metastatic disease after nCRT, poor tumor visibility on DWMRI, or withdrawal from study participation. The primary diagnostic workup consisted of an endoscopy with biopsy for diagnosis, as well as an integrated ${ }^{18} \mathrm{~F}-\mathrm{FDG}$ PET/CT scan for clinical staging. 


\section{Treatment}

The neoadjuvant treatment regimen consisted of weekly intravenous administration of carboplatin and paclitaxel for 5 weeks with concurrent radiotherapy (41.4 Gy in 23 fractions of $1.8 \mathrm{~Gy}$, see Supplementary material for details) [2]. Surgical resection consisted of a transthoracic or transhiatal esophagectomy with en-bloc two-field lymphadenectomy and gastric conduit reconstruction with either cervical or intrathoracic anastomosis.

\section{Histopathological assessment}

The surgical resection specimen was assessed by a specialized gastrointestinal pathologist who was blinded for the results of the DW-MRI scans. Patients were staged in accordance with the $7^{\text {th }}$ edition of the Union for International Cancer Control (UICC) [30]. Pathologic tumor regression was reported according to the Mandard system (tumor regression grade [TRG] 1 [pCR, ypT0]: complete response with absence of residual cancer cells; TRG 2: rare residual cancer cells scattered through fibrosis; TRG 3: increase in the number of residual cancer cells, but fibrosis still predominates; TRG 4: residual cancer outgrowing fibrosis; TRG 5: absence of regressive changes) [31]. In the absence of macroscopic tumor, any abnormally appearing tissue was evaluated in order to make an adequate assessment of the presence of residual tumor and the effects of therapy.

\section{Image acquisition}

Patients underwent six sequential MRI scans. One baseline MRI scan was performed at a median of 5 days (interquartile range [IQR] 4-8 days) prior to nCRT in addition to the conventional diagnostic workup. Subsequently, five additional MRI scans were performed weekly during nCRT (see Supplementary Fig. 1 for the study design).

All images were acquired on a 1.5-T Philips Ingenia. Respiratory-triggered transverse anatomical T2-weighted scans (tT2W) and DW-MRI scans with three $b$-values ( 0 , 200 , and $800 \mathrm{~s} / \mathrm{mm}^{2}$ ) were acquired in coronal planes (see Supplementary methods for details).

\section{Image analysis}

The primary tumor - excluding the lumen-was delineated based on the signal of the baseline DW-MRI scans with a $b$-value of $800 \mathrm{~s} / \mathrm{mm}^{2}$ using open source software with a semiautomated delineation method (ITK-SNAP, www.itksnap.org) $[32,33]$, allowing for manual editing by two readers (A.S.B. and S.E.H.) in consensus. The primary contouring was propagated to the DW-MRI scans of the subsequent weeks, followed by manual adjustment by one reader (A.S.B.) based on signal reduction on the b800 DW-MRI scans and tumor regression on the tT2W scans using in-house-developed delineation software [34]. Contouring was performed conservatively to avoid edges of the tumor boundaries, as ADC values at the boundaries might be unreliable due to motion or image distortions [15]. In all cases, the apparent tumor bed was verified based on the tT2W images. Since the in-house-developed delineation software interprets images as $3 \mathrm{D}$ volumes, there was no need to generate multiplanar reconstructions. The readers were blinded to patient-related characteristics and clinical outcome in terms of pathologic response.

ADC maps were generated for each slice based on a monoexponential model fitted on $b$-values of 0,200 , and 800 $\mathrm{s} / \mathrm{mm}^{2}$, as based on earlier experience $[14,15]$. Mean tumor ADC values were extracted from the DW-MRI volume of interests. The relative changes in mean ADC values between the baseline scans and the scans during nCRT were calculated and included in the analyses, as based on previous literature $\left(\triangle \mathrm{ADC}(\%)=\left[\right.\right.$ mean $\mathrm{ADC}_{\mathrm{week}(n)}-$ mean $\left.\mathrm{ADC}_{\text {baseline }}\right] /$ mean $\left.\mathrm{ADC}_{\text {baseline }}\right)[14,15,24]$.

\section{Statistical analysis}

Patient and treatment-related characteristics are described as counts with percentages, mean ( \pm standard deviation $[\mathrm{SD}]$ ), or median (IQR). The median delineated tumor volume on the baseline DW-MRI scan was compared between patients with a pCR and non-pCR using the nonparametric Mann-Whitney $U$ test.

In order to determine the optimal timing of DW-MRI scanning for prediction of $\mathrm{pCR}$, the $\triangle \mathrm{ADC}(\%)$ was compared between patients with a pCR and non-pCR per week using the Mann-Whitney $U$ test. The ability of the $\triangle \mathrm{ADC}(\%)$ parameters per week to discriminate between $\mathrm{pCR}$ and non-pCR was quantified using ridge regression, including tumor histology as determined on the tumor biopsy - an important known factor to impact pCR. Subsequently, the area under the receiver-operating characteristic (ROC) curve ( $c$-statistic) was calculated. Missing ADC values were imputed with using multiple imputation. Subsequently, $\triangle \mathrm{ADC}(\%)$ values were calculated and the ridge regression model was fitted on all imputed datasets (see Supplementary material for details).

Furthermore, in order to determine whether the results of future studies could be optimized when applying additional exclusion criteria, a post hoc sensitivity analysis was performed. Patients with small tumor volumes (as the signal blurring caused by respiratory motion is more pronounced in small tumors) as well as patients with a histologic tumor type other than adenocarcinoma or squamous cell carcinoma based on the resection specimen were excluded. The performance measure of interest in this sensitivity analysis was the $c$-statistic. 
All statistical analyses were performed using R software for statistical computing version 3.5.1 ('mice' [35], 'glmnet' [36], and 'Hmisc' [37] packages, www.R-project.org). The significance level was set at $p<0.05$. No corrections for multiple testing were performed, as the universal null hypothesis was not of interest [38]. Furthermore, as this study was of descriptive nature, no formal power calculation was performed.

\section{Results}

\section{Patients}

A total of 32 patients with newly diagnosed esophageal cancer were enrolled in the prospective study. Of these patients, 8 were excluded for the current analysis based on unexpected distant metastatic disease after nCRT $(n=2)$, tumor histology other than squamous cell carcinoma or adenocarcinoma as based on the primary tumor biopsy $(n=2)$, poor tumor visibility on DWMRI $(n=3)$, or withdrawal from study participation $(n=1)$ (Supplementary Fig. 2). The final study population comprised 24 patients with a mean age of 65 years ( \pm 8 years) and all but 2 were male $(92 \%)$. The majority of the patients had an adenocarcinoma (67\%). Median time between nCRT and esophagectomy was 10 weeks (IQR 7-14 weeks). A pCR (TRG 1) after nCRT was observed in 7 patients (29\%). Table 1 gives an overview of the clinical characteristics of the study population.

All patients received 5 cycles of chemotherapy and the full course of radiation therapy. In one patient, it was decided during nCRT to extend the regimen with 1 week, to a total dose of 50.4 Gy and 6 cycles of chemotherapy. Pretreatment DW-MRI scans were available in all patients. Two DW-MRI scans during nCRT were missing due to patient's refusal $(n=1)$ or image acquisition problems $(n=1)$, resulting in a total of 142 MRI scans.

The median delineated tumor volume on the baseline DWMRI scan was $15 \mathrm{~mL}$ (IQR 11-23 mL) and did not significantly differ between pCR and non-pCR patients (median [IQR]: $11 \mathrm{~mL}$ [7-22 $\mathrm{mL}$ ] versus $16 \mathrm{~mL}$ [11-23 mL], respectively, $p=0.318$ ).

\section{ADC changes during $\mathrm{nCRT}$}

The relative increase in tumor ADC from baseline DW-MRI scans to scans acquired in the second week of nCRT $\left(\triangle \mathrm{ADC}_{\text {week } 2}\right.$ ) was significantly associated with $\mathrm{pCR}$ (median [IQR]: $36 \%$ [30-41\%] for pCR versus 16\% [14-29\%] for non-pCR, $p=0.004)$. In contrast, relative changes in ADC from baseline to DW-MRI scans acquired in the other weeks of nCRT were not significantly different between pCR and non-pCR groups (Table 2, Fig. 1). Figure 2 presents baseline
MRI scans and MRI scans in the second week of nCRT of a patient with $\mathrm{pCR}$.

ROC curve analyses after ridge-penalized regression analyses taking histology into account demonstrated the highest $c$-statistic of 0.87 for the relative ADC increase from baseline to week 2 of nCRT ( $\triangle \mathrm{ADC}_{\text {week } 2}$ ) combined with histology. Poorer discriminative ability was observed for histology alone ( $c$-statistic 0.67 ) or histology in combination with the $\triangle \mathrm{ADC}(\%)$ of the other weeks (c-statistics $0.73-0.80$, Table 3, Fig. 3). A predictive probability plot for $\mathrm{pCR}$ based on $\triangle \mathrm{ADC}(\%)$ from baseline to week 2 for squamous cell carcinomas and adenocarcinomas is presented in Fig. 4, demonstrating an increase in the probability for $\mathrm{pCR}$ when $\triangle \mathrm{ADC}(\%)$ increases.

\section{Sensitivity analyses}

For the post hoc sensitivity analysis, an additional 4 patients were excluded based on a delineated tumor volume on the baseline DW-MRI scan $<7 \mathrm{~mL}(n=3)$ and tumor histology other than adenocarcinoma or squamous cell carcinoma as based on the resection specimen $(n=1)$. In this study population of 20 patients, 5 patients had a pCR of which 2 had an adenocarcinoma and 3 had a squamous cell carcinoma. Exclusion of these additional 4 patients resulted in significant differences between patients with $\mathrm{pCR}$ and non-pCR in $\triangle \mathrm{ADC}(\%)$ from baseline to weeks 2,4 , and 5 (Table 2). Furthermore, the $c$-statistics improved for the regression models with $\triangle \mathrm{ADC}(\%)$ of weeks $1,2,3,4$, and 5 to 0.84 , $0.97,0.77,0.93$, and 0.90 , respectively (Table 3 ).

Multiple imputation of the missing ADC value of week 4 and week 5 did not substantially impact the results of the regression analysis in terms of the observed $c$-statistics in the entire cohort ( 0.80 and 0.72 in the imputed datasets compared to 0.79 and 0.75 in the complete case datasets, respectively) or in the sensitivity analysis ( 0.93 and 0.87 in the imputed dataset compared to 0.93 and 0.90 in the complete case dataset, respectively) (Supplementary Table 2).

\section{Discussion}

This prospective study was designed to assess the optimal timing of DW-MRI scanning during nCRT for prediction of $\mathrm{pCR}$ in esophageal cancer patients. The relative change in $\mathrm{ADC}(\triangle \mathrm{ADC})$ during the first 2 weeks of $\mathrm{nCRT}$ demonstrated the highest predictive ability for $\mathrm{pCR}$ at the time of surgery. This is of important clinical value as early response evaluation could enable individualized treatment regimens. Accurate assessment of response to nCRT is not only important for safe implementation of an organ-sparing approach in patients with $\mathrm{pCR}$, but also to adapt treatment strategies in expected poor responders. Improving the accuracy of response evaluation after nCRT may provide improved outcomes for both patient groups. 
Table 1 Clinical characteristics of the study population

\begin{tabular}{|c|c|c|c|c|c|c|}
\hline \multirow[t]{2}{*}{ Characteristic } & \multicolumn{2}{|c|}{ Full cohort $(n=24)$} & \multicolumn{2}{|c|}{$\mathrm{pCR}(n=7)$} & \multicolumn{2}{|c|}{ Non-pCR $(n=17)$} \\
\hline & $n$ & $(\%)$ & $n$ & $(\%)$ & $n$ & $(\%)$ \\
\hline Age at diagnosis, in years $($ mean $\pm \mathrm{SD})$ & \multicolumn{2}{|c|}{$65 \pm 8$} & \multicolumn{2}{|l|}{$68 \pm 7$} & \multicolumn{2}{|c|}{$64 \pm 8$} \\
\hline \multicolumn{7}{|l|}{ Sex } \\
\hline Male & 22 & $92 \%$ & 7 & $100 \%$ & 15 & $88 \%$ \\
\hline Female & 2 & $8 \%$ & 0 & $0 \%$ & 2 & $12 \%$ \\
\hline \multicolumn{7}{|l|}{ Tumor location } \\
\hline Middle esophagus & 1 & $4 \%$ & 0 & $0 \%$ & 1 & $6 \%$ \\
\hline Distal esophagus & 22 & $92 \%$ & 7 & $100 \%$ & 15 & $88 \%$ \\
\hline Gastroesophageal junction & 1 & $4 \%$ & 0 & $0 \%$ & 1 & $6 \%$ \\
\hline \multicolumn{7}{|l|}{ Clinical T stage* } \\
\hline $\mathrm{cT} 2$ & 1 & $4 \%$ & 0 & $0 \%$ & 1 & $6 \%$ \\
\hline cT3 & 23 & $96 \%$ & 7 & $100 \%$ & 16 & $94 \%$ \\
\hline \multicolumn{7}{|l|}{ Clinical N stage* } \\
\hline cNO & 8 & $33 \%$ & 3 & $43 \%$ & 5 & $29 \%$ \\
\hline $\mathrm{cN} 1$ & 13 & $54 \%$ & 2 & $29 \%$ & 11 & $65 \%$ \\
\hline $\mathrm{cN} 2$ & 2 & $8 \%$ & 2 & $29 \%$ & 0 & $0 \%$ \\
\hline $\mathrm{cN} 3$ & 1 & $4 \%$ & 0 & $0 \%$ & 1 & $6 \%$ \\
\hline \multicolumn{7}{|l|}{ Histologic tumor type (biopsy) } \\
\hline Adenocarcinoma & 16 & $67 \%$ & 3 & $43 \%$ & 13 & $76 \%$ \\
\hline Squamous cell carcinoma & 8 & $33 \%$ & 4 & $57 \%$ & 4 & $24 \%$ \\
\hline \multicolumn{7}{|l|}{ Tumor regression grade (TRG) } \\
\hline TRG 1 (pCR) & 7 & $29 \%$ & 7 & $100 \%$ & NA & \\
\hline TRG 2 & 6 & $25 \%$ & NA & & 6 & $35 \%$ \\
\hline TRG 3 & 7 & $29 \%$ & NA & & 7 & $41 \%$ \\
\hline TRG 4 & 3 & $13 \%$ & NA & & 3 & $18 \%$ \\
\hline TRG 5 & 1 & $4 \%$ & NA & & 1 & $6 \%$ \\
\hline \multicolumn{7}{|l|}{ Pathological T stage* } \\
\hline урТ0 & 7 & $29 \%$ & 7 & $100 \%$ & NA & \\
\hline урT1 & 3 & $13 \%$ & NA & & 3 & $18 \%$ \\
\hline урТ2 & 6 & $25 \%$ & NA & & 6 & $35 \%$ \\
\hline урT3 & 8 & $33 \%$ & NA & & 8 & $47 \%$ \\
\hline \multicolumn{7}{|l|}{ Pathological N stage* } \\
\hline ypN0 & 15 & $63 \%$ & 6 & $86 \%$ & 9 & $53 \%$ \\
\hline ypN1 & 4 & $17 \%$ & 1 & $14 \%$ & 3 & $18 \%$ \\
\hline ypN2 & 4 & $17 \%$ & 0 & $0 \%$ & 4 & $24 \%$ \\
\hline ypN3 & 1 & $4 \%$ & 0 & $0 \%$ & 1 & $6 \%$ \\
\hline \multicolumn{7}{|l|}{ Surgical approach } \\
\hline Thoracolaparoscopic & 18 & $75 \%$ & 5 & $71 \%$ & 13 & $76 \%$ \\
\hline Laparoscopic transhiatal & 5 & $21 \%$ & 1 & $14 \%$ & 4 & $24 \%$ \\
\hline Open transthoracic & 1 & $4 \%$ & 1 & $14 \%$ & 0 & $0 \%$ \\
\hline Lymph node yield (median, IQR) & 30 & $(19-36)$ & 29 & $(23-40)$ & 30 & $(19-35)$ \\
\hline Positive lymph nodes harvested (median, IQR) & 0 & $(0-1)$ & 0 & $(0-0)$ & 1 & $(0-3)$ \\
\hline
\end{tabular}

$I Q R$ interquartile range; $N A$ not applicable; $p C R$ pathologic complete response; $S D$ standard deviation; TRG tumor regression grade

*Clinical and histopathologic T- and N-stage are based on UICC TNM 7th edition

In order to improve patient-friendliness of disease monitoring and (re)staging procedures, it is important to minimize the burden of these procedures. As demonstrated before, DW-
MRI is generally well-tolerated by patients, although shorter acquisition times as well as altered body positioning could further improve patient experience [39]. Our results could 
Table 2 Relative changes in ADC per week during neoadjuvant chemoradiotherapy between esophageal cancer patients with a pathologic complete response and non-pathologic complete response

\begin{tabular}{|c|c|c|c|c|}
\hline & & \multicolumn{2}{|c|}{ Median $\triangle \mathrm{ADC}(\%)(\mathrm{IQR})$} & \multirow[t]{2}{*}{$p$ value* } \\
\hline & & $\mathrm{pCR}$ & Non-pCR & \\
\hline \multirow[t]{5}{*}{ Full cohort $(n=24)$} & Week 1 & $13(5,23)$ & $5(-2,19)$ & 0.260 \\
\hline & Week 2 & $36(30,41)$ & $16(14,29)$ & 0.004 \\
\hline & Week 3 & $34(24,69)$ & $30(17,42)$ & 0.318 \\
\hline & Week 4 & $52(37,64)$ & $35(26,47)$ & 0.065 \\
\hline & Week 5 & $58(34,83)$ & $40(27,53)$ & 0.198 \\
\hline \multirow[t]{5}{*}{ Sensitivity analyses $(n=20)^{\dagger}$} & Week 1 & $13(11,23)$ & $5(-2,19)$ & 0.098 \\
\hline & Week 2 & $37(24,41)$ & $16(10,19)$ & 0.001 \\
\hline & Week 3 & $42(31,69)$ & $27(5,42)$ & 0.168 \\
\hline & Week 4 & $63(52,64)$ & $34(24,46)$ & 0.002 \\
\hline & Week 5 & $59(58,83)$ & $38(25,50)$ & 0.003 \\
\hline
\end{tabular}

$A D C$ apparent diffusion coefficient; $I Q R$ interquartile range; $p C R$ pathologic complete response

* $p$ value based on Mann-Whitney $U$ test

${ }^{\dagger}$ After post-hoc exclusion of additional four patients based on baseline tumor volume delineated on DW-MRI $<7 \mathrm{~mL}(n=3)$ and tumor histology other than adenocarcinoma or squamous cell carcinoma as based on the resection specimen $(n=1)$

further aid the minimization of the burden of repeated scanning for patients, as well as optimal usage of the available (financial) resources, while assuring the best predictive ability of DW-MRI.

Previous studies focusing on DW-MRI scanning in response prediction for esophageal cancer have mostly demonstrated promising findings regarding the predictive value of $\triangle \mathrm{ADC}(\%)$ for response prediction to nCRT $[14,15,18,40$, 41]. The majority of these studies reported significant differences between responders and nonresponders in $\triangle \mathrm{ADC}(\%)$ from baseline to during nCRT $[14,15,24,41]$, whereas others report significant differences in $\triangle \mathrm{ADC}(\%)$ from baseline to follow-up after nCRT $[18,41]$. Nevertheless, not all studies found the same predictive effect size, nor did they all report a significant relation between $\triangle \mathrm{ADC}(\%)$ and response $[42,43]$. Several factors may account for these differences. First, ADCs were calculated based on different $b$-values varying from 0 and 1000 [41], 0 and 600 [24] to 0,200 , and 800 [14, 15]. The choice of $b$-values and the number of signal averages (NSAs) per $b$-value, as well as the calculation of ADC values by various modeling strategies, are known to impact tumor ADC estimates [21]. Second, delineation methods for

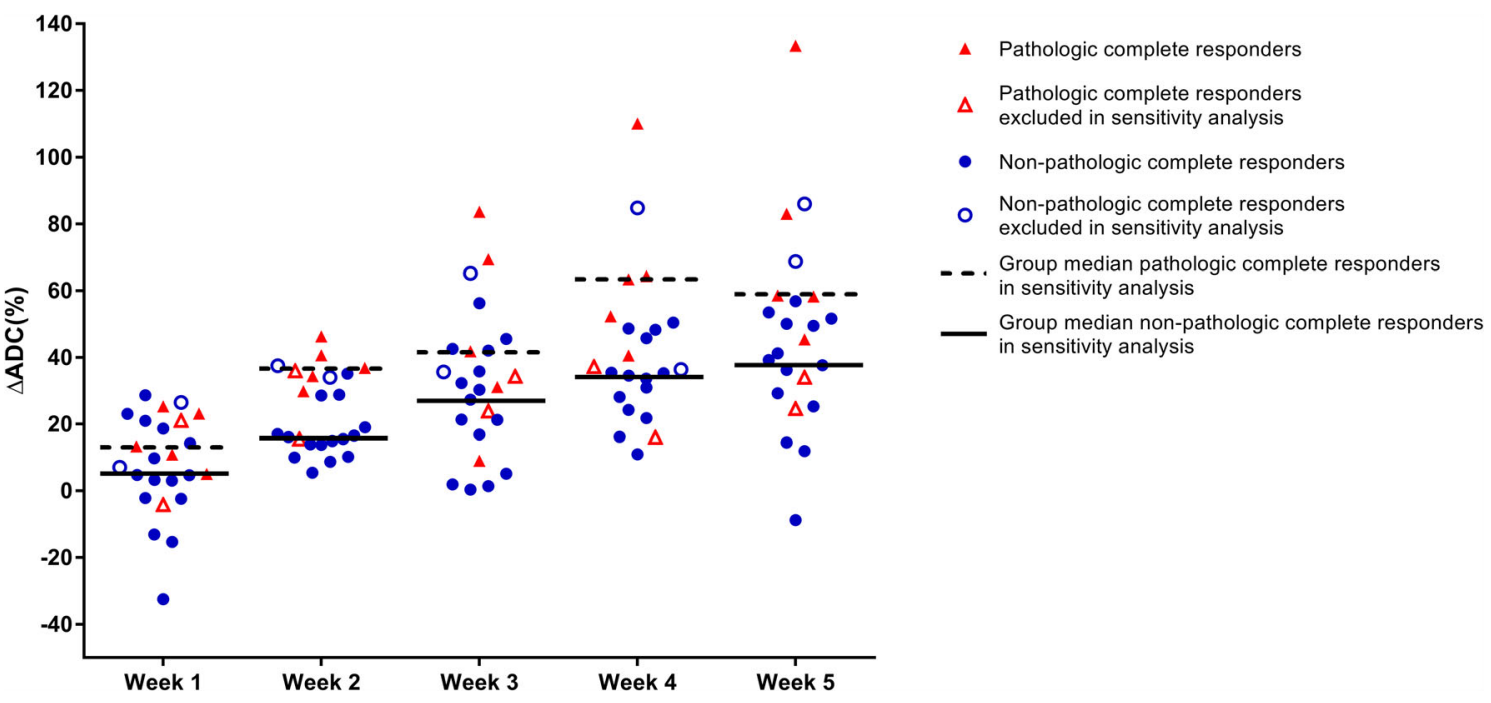

Fig. 1 Relative changes in ADC values between baseline scans and scans during neoadjuvant chemoradiotherapy between pathologic complete responders (pCR, red triangles) and poor responders (non-pCR, blue

circles). Patients who were excluded in the post hoc sensitivity analysis are marked with an empty symbol. 
Fig. 2 Patient with a cT3N2M0 distal esophageal squamous cell carcinoma with a pathologic complete response (pCR, TRG 1) to neoadjuvant

chemoradiotherapy and a $\triangle \mathrm{ADC}_{\text {week } 2}$ of $29 \%$. T2 weighted images (a and b), diffusion-weighted images $(b$ value $\left.=800 \mathrm{~s} / \mathrm{mm}^{2}\right)(\mathbf{c}$ and $\mathbf{d})$, and ADC maps (e and f) on a 1.5-T MR scanner before (a, c, e) and in the second week of neoadjuvant chemoradiotherapy (b, d, f)

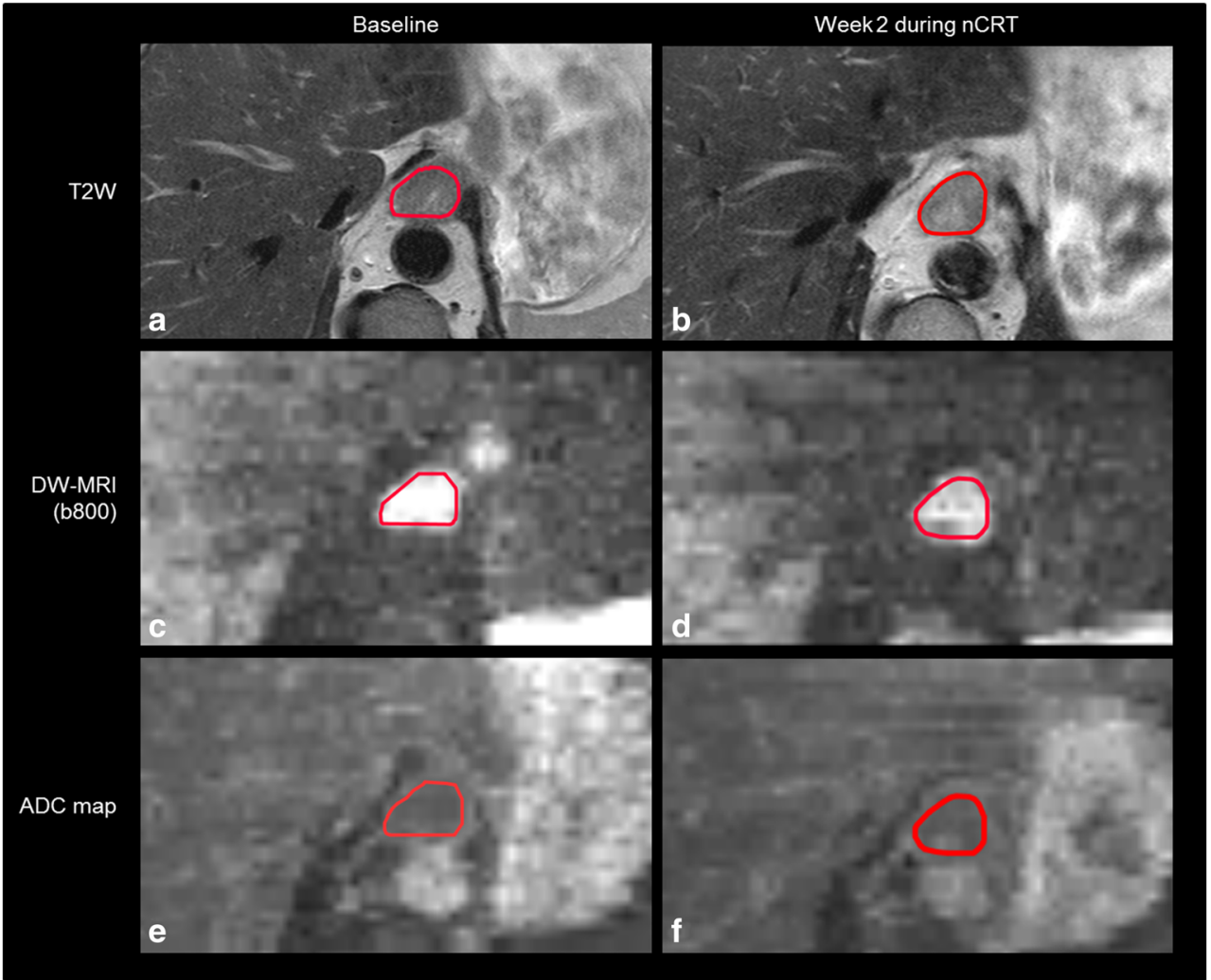

determining the volume of interest differ. Some studies consider the entire tumor volume as volume of interest $[14,15$, 18], whereas others only delineated the tumor on the most representative tumor slice [41]. Lastly, the nCRT regimen of choice varied between the studies.

Similar to our study, Wang et al [24] performed weekly DW-MRI scanning during CRT in esophageal cancer patients to determine the optimal timing of response evaluation with DW-MRI. They also demonstrated DW-MRI scanning in the second or third week of CRT to be optimal for response assessment. As their study included only esophageal squamous cell carcinomas, used imaging response criteria as a reference standard (i.e., RECIST criteria [44] that assessed 52\% of the patients to be a complete responder) instead of histopathology, used a different nCRT regimen (chemotherapy consisting of cisplatin with either 5-fluorouracil or paclitaxel and radiotherapy consisting of 60 Gy in 30 fractions), and did not use $\triangle \mathrm{ADC}(\%)$ but only single time point $\mathrm{ADC}$ values as measured, similar findings might not have been expected. Additionally, earlier studies by our group as well as the University of Texas MD Anderson Cancer Center that performed DW-MRI in the second or third week found these $\triangle \mathrm{ADC}(\%)$ values to be highly predictive of response to nCRT $[14,15]$. Together, this supports the robustness of the findings from the current study.

The interobserver reproducibility of tumor delineation on DW-MRI and ADC measurements in esophageal cancer was shown to be very good by two previous studies, especially for the semi-automated volumetric measurement method (intraclass correlation coefficient $0.96,95 \%$ CI $0.91-0.98, p<0.001$ ), which was also applied in the current study [15, 43]. One of these studies compared manual delineation of a region of interest on the most representative tumor slice to semi-automatic delineation of the whole tumor volume and found negligible differences in mean ADC measurements (between -0.25 and $0.31 \%$ ) [15]. A voxel-based analytical method, where changes in individual voxels can be monitored, may even provide more reliable results [43]. However, such an approach is challenging, since tumor regression is observed during nCRT and the esophagus is a moving organ [28], making spatial registration of DWMRI obtained before and after start of nCRT complicated.

To improve external validity of our results, no cutoff values for $\mathrm{ADC}$ or $\triangle \mathrm{ADC}$ measurements for classification of complete responders versus nonresponders are reported in our study. Cutoff values are likely to be highly influenced by delineation techniques and determination of a $2 \mathrm{D}$ or $3 \mathrm{D}$ region of interest, as well as the $b$-values on which ADCs are calculated. To demonstrate this, we highlighted previously reported significant cutoff values for $\triangle \mathrm{ADC}(\%), \mathrm{ADC}_{\text {mean }}$, and $\mathrm{ADC}_{\text {median }}$ for discrimination of responders versus nonresponders in the data of the current study in Supplementary Fig. 3.

A recent meta-analysis demonstrated a pooled AUC of 0.91 (95\% CI 0.89-0.94) of $\triangle \mathrm{ADC}(\%)$ values for treatment response prediction in esophageal cancer based on four studies 
Table 3 Ridge regression analyses demonstrating the discriminatory value of DW-MRI parameters per week with pathologic complete response (TRG 1) as outcome variable

\begin{tabular}{|c|c|c|c|c|c|c|}
\hline \multirow[t]{2}{*}{ Intercept and predictors } & \multicolumn{3}{|c|}{ Full cohort $(n=24)$} & \multicolumn{3}{|c|}{ Sensitivity analyses $(n=20)^{*}$} \\
\hline & $\beta$ & OR & $c$-statistic & $\beta$ & OR & $c$-statistic \\
\hline \multicolumn{7}{|l|}{ Histology } \\
\hline Intercept & -1.00 & & 0.67 & -1.28 & & 0.67 \\
\hline Squamous cell carcinoma $^{\dagger}$ & 0.33 & 1.39 & & 0.49 & 1.63 & \\
\hline \multicolumn{7}{|l|}{ Week 1} \\
\hline Intercept & -1.74 & & 0.80 & -2.30 & & 0.84 \\
\hline$\Delta \mathrm{ADC}_{\text {week } 1}(\%)$ & 0.04 & 1.04 & & 0.06 & 1.06 & \\
\hline Squamous cell carcinoma ${ }^{\dagger}$ & 1.29 & 3.63 & & 1.44 & 4.24 & \\
\hline \multicolumn{7}{|l|}{ Week 2} \\
\hline Intercept & -3.45 & & 0.87 & -5.17 & & 0.97 \\
\hline$\Delta \mathrm{ADC}_{\text {week } 2}(\%)$ & 0.09 & 1.09 & & 0.15 & 1.16 & \\
\hline Squamous cell carcinoma $^{\dagger}$ & 0.89 & 2.44 & & 0.32 & 1.37 & \\
\hline \multicolumn{7}{|l|}{ Week 3} \\
\hline Intercept & -1.09 & & 0.73 & -1.76 & & 0.77 \\
\hline$\Delta \mathrm{ADC}_{\text {week } 3}(\%)$ & 0.00 & 1.00 & & 0.02 & 1.02 & \\
\hline Squamous cell carcinoma ${ }^{\dagger}$ & 0.20 & 1.23 & & 0.44 & 1.56 & \\
\hline \multicolumn{7}{|l|}{ Week 4} \\
\hline Intercept & -1.61 & & 0.79 & -4.76 & & 0.93 \\
\hline$\Delta \mathrm{ADC}_{\text {week } 4}(\%)$ & 0.01 & 1.01 & & 0.08 & 1.08 & \\
\hline Squamous cell carcinoma ${ }^{\dagger}$ & 0.50 & 1.65 & & 0.16 & 1.18 & \\
\hline \multicolumn{7}{|l|}{ Week 5} \\
\hline Intercept & -1.57 & & 0.75 & -4.03 & & 0.90 \\
\hline$\Delta \mathrm{ADC}_{\text {week } 5}(\%)$ & 0.01 & 1.01 & & 0.06 & 1.06 & \\
\hline Squamous cell carcinoma ${ }^{\dagger}$ & 0.69 & 1.99 & & 0.64 & 1.90 & \\
\hline
\end{tabular}

Note: Due to rounding, the reported odds ratios might not precisely correspond with the reported beta regression coefficients $A D C$ apparent diffusion coefficient, $c$-statistic concordance statistic, $O R$ odds ratio, $p C R$ pathologic complete response

*After post hoc exclusion of additional four patients based on baseline tumor volume delineated on DW-MRI $<7 \mathrm{~mL}(n=3)$ and tumor histology other than adenocarcinoma or squamous cell carcinoma as based on the resection specimen $(n=1)$

${ }^{\dagger}$ Adenocarcinoma was used as reference category

[40]. We were not able to reproduce these results in our full cohort, but this cohort included three patients with small tumor volumes $(<7 \mathrm{~mL})$ and one patient who had a mixed adenoneuroendocrine carcinoma (MANEC) upon histopathological evaluation of the resection specimen. As MANECs are known to respond well to nCRT during treatment (which is reflected by an increase in ADC in the first weeks) but progress rapidly after completion, the inclusion of this patient clearly influenced the obtained results in this relatively small cohort $[45,46]$. Exclusion of the aforementioned patients dramatically improved the performance of $\triangle \mathrm{ADC}(\%)$ for pCR prediction, resulting in a $c$-statistic of 0.97 for week 2 . This also demonstrated that the predictive value of relative changes in ADC seems decreased for small tumors, which might be explained by the respiratory movement of the tumor during the DW-MRI scan. Typically, respiratory motion amplitudes of $1-2 \mathrm{~cm}$ are observed during scanning [28, 47], which might negatively impact the quantitative ADC assessment especially in small tumors. Improvement in pCR prediction in future studies may be obtained by incorporating motion management techniques.

Previous work has also demonstrated additional value of DCE-MRI, as well as PET-CT scanning in the prediction of treatment response in esophageal cancer patients [15, 48-52]. Furthermore, emerging biomarkers such as circulating tumor DNA might further improve the predictive performance and might contribute to the safe investigation of an organ-sparing approach for predicted $\mathrm{pCR}$ to $\mathrm{nCRT}$.

Significant strengths of the current study include the consistent use of one nCRT regimen for all patients, the presence of a histopathologic reference standard in all patients, the inclusion of both squamous cell carcinomas and adenocarcinomas, and the consistent delineation by semi-automatic contouring. However, the specific hardware characteristics of the MR scanner and scan sequences, as well as the applied 
Fig. 3 Receiver-operating characteristic curve analysis for the regression models with relative changes in ADC per week, as well as histopathological tumor type, for discriminating between pCR and non-pCR patients in the full cohort (a) as well as in the sensitivity analysis (b)
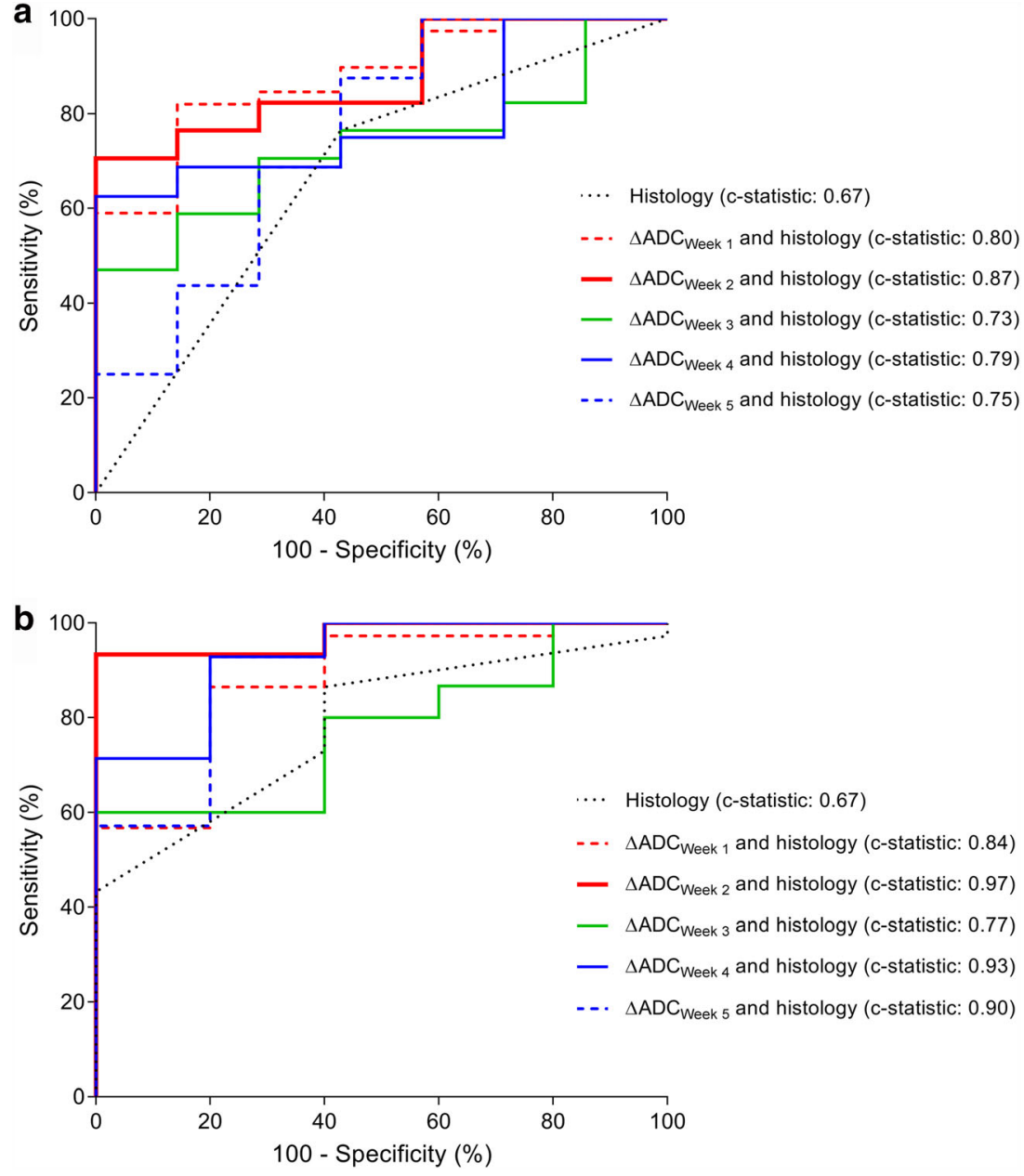

delineation technique and calculation of the ADC map by a mono-exponential model based on three $b$-values, may limit the generalizability of the results. In addition, whole-tumor summary statistics (such as the mean ADC) are easily applicable, but fail to fully address the important issue of tumor heterogeneity [21]. Furthermore, the relatively small study population might have led to false-negative results (type II error) for differences in $\triangle \mathrm{ADC}(\%)$ between responders and nonresponders from baseline
Fig. 4 Predictive probability plot for pathologic complete response (pCR) based on the fitted regression model including relative changes in $\mathrm{ADC}$ $(\triangle \mathrm{ADC}(\%))$ from baseline to week 2 for squamous cell carcinomas (blue) and adenocarcinomas (red). The dashed lines represent the probability plot for the sensitivity analysis after additional exclusion of 4 patients

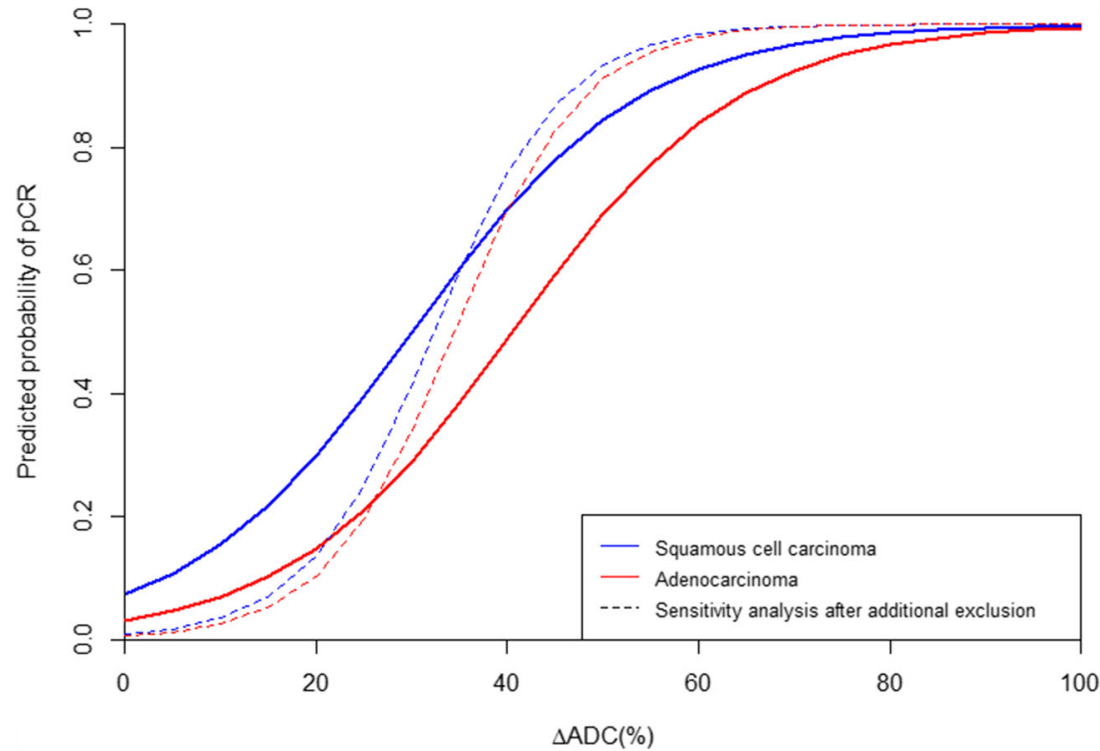


to the other weeks than week 2. Moreover, patients were included during a rather long study period of 2.5 years, since many eligible patients refused participation in this demanding and timeconsuming study with weekly MRI scanning. However, the patients generally tolerated the MRI scans well (only 3 patients canceled 1 MRI scan during their treatment in the entire cohort of 32 patients) and no adverse events occurred. Lastly, DW-MRI scanning is currently not routinely used in the staging of patients with esophageal cancer, which challenges the direct implementation of the results in clinical practice.

Future comparative studies should focus on further improving response evaluation after nCRT. In this regard, the recently started Dutch multicenter PRIDE study will further investigate the findings of the DW-MRI pilot studies in a larger patient cohort and aims at developing a multimodal clinically applicable prediction model [53].

In conclusion, treatment-induced change in tumor ADC as measured on DW-MRI during the second week is most predictive for $\mathrm{pCR}$ to $\mathrm{nCRT}$ in esophageal squamous cell carcinoma and adenocarcinoma.

Funding information The authors state that this work has not received any funding.

\section{Compliance with ethical standards}

Guarantor The scientific guarantor of this publication is Gert J. Meijer (g.j.meijer@umcutrecht.nl).

Conflict of interest The authors declare that they have no conflict of interest.

Statistics and biometry Three of the authors have significant statistical expertise: A.S. Borggreve, L. Goense, and P.S.N. van Rossum hold a postgraduate master's degree in epidemiology from Utrecht University, specializing in clinical epidemiology and medical statistics.

Informed consent Written informed consent was obtained from all patients in this study.

Ethical approval Institutional Review Board approval was obtained.

Study subjects or cohorts overlap Some study subjects or cohorts have been previously reported in Heethuis et al (Quantification of variations in intra-fraction motion of esophageal tumors over the course of neoadjuvant chemoradiotherapy based on cine-MRI. Phys Med Biol.;63(14):145019) and Boekhoff et al (PO-0951: what CTV-to-PTV margins are required for esophageal cancer radiotherapy? Radiother Oncol. 2018;127:S519-S520). The current study was a prespecified secondary analysis and reported on a completely different outcome (histopathological tumor regression) and used different MR scans (DW-MRI instead of anatomical T2W MRI) than the previous studies.

\footnotetext{
Methodology

- prospective

- observational study

- performed at one institution
}

Open Access This article is distributed under the terms of the Creative Commons Attribution 4.0 International License (http:// creativecommons.org/licenses/by/4.0/), which permits unrestricted use, distribution, and reproduction in any medium, provided you give appropriate credit to the original author(s) and the source, provide a link to the Creative Commons license, and indicate if changes were made.

\section{References}

1. Lordick F, Mariette C, Haustermans K, Obermannová R, Arnold D (2016) Oesophageal cancer: ESMOClinical Practice Guidelines for diagnosis, treatment and follow-up. Ann Oncol 27:v50-v57. https://doi.org/10.1093/annonc/mdw329

2. van Hagen P, Hulshof MCCM, van Lanschot JJB et al (2012) Preoperative chemoradiotherapy for esophageal or junctional cancer. N Engl J Med 366:2074-2084. https://doi.org/10.1056/ NEJMoa1112088

3. Sjoquist KM, Burmeister BH, Smithers BM et al (2011) Survival after neoadjuvant chemotherapy or chemoradiotherapy for resectable oesophageal carcinoma: an updated meta-analysis. Lancet Oncol 12:681-692. https://doi.org/10.1016/S1470-2045(11) 70142-5

4. Shapiro J, Van Lanschot JJB, Hulshof MCCM et al (2015) Neoadjuvant chemoradiotherapy plus surgery versus surgery alone for oesophageal or junctional cancer (CROSS): long-term results of a randomised controlled trial. Lancet Oncol 16:1090-1098. https:// doi.org/10.1016/S1470-2045(15)00040-6

5. Steffen T, Dietrich D, Schnider A et al (2019) Recurrence patterns and long-term results after induction chemotherapy, chemoradiotherapy, and curative surgery in patients with locally advanced esophageal cancer. Ann Surg 269:83-87. https://doi.org/10.1097/ SLA.0000000000002435

6. Walsh TN, Noonan N, Hollywood D et al (1996) A comparison of multimodal therapy and surgery for esophageal adenocarcinoma. $\mathrm{N}$ Engl J Med 335:462-467. https://doi.org/10.1056/ NEJM199608153350702

7. Burmeister BH, Smithers BM, Gebski V et al (2005) Surgery alone versus chemoradiotherapy followed by surgery for resectable cancer of the oesophagus: a randomised controlled phase III trial. Lancet Oncol 6:659-668. https://doi.org/10.1016/S1470-2045(05) 70288-6

8. Djärv T, Lagergren J, Blazeby JM, Lagergren P (2008) Long-term health-related quality of life following surgery for oesophageal cancer. Br J Surg 95:1121-1126. https://doi.org/10.1002/bjs.6293

9. Mc Cormack O, Zaborowski A, King S et al (2014) New-onset atrial fibrillation post-surgery for esophageal and junctional cancer incidence, management, and impact on short- and long-term outcomes. Ann Surg 260:772-778. https://doi.org/10.1097/SLA. 0000000000000960

10. Kassis ES, Kosinski AS, Ross P Jr, Koppes KE, Donahue JM, Daniel VC (2013) Predictors of anastomotic leak after esophagectomy: an analysis of the Society of Thoracic Surgeons General Thoracic Database. Ann Thorac Surg 96:1919-1926. https://doi. org/10.1016/j.athoracsur.2013.07.119

11. Busweiler LAD, Wijnhoven BPL, van Berge Henegouwen MI et al (2016) Early outcomes from the Dutch Upper Gastrointestinal Cancer Audit. Br J Surg 103:1855-1863. https://doi.org/10.1002/ bjs. 10303

12. Schandl A, Lagergren J, Johar A, Lagergren P (2016) Healthrelated quality of life 10 years after oesophageal cancer surgery. Eur J Cancer 69:43-50. https://doi.org/10.1016/J.EJCA.2016.09.032 
13. Noordman BJ, Wijnhoven BPL, Lagarde SM et al (2017) Active surveillance in clinically complete responders after neoadjuvant chemoradiotherapy for esophageal or junctional cancer. Dis Esophagus 30:1-8. https://doi.org/10.1093/dote/dox100

14. van Rossum PS, van Lier AL, van Vulpen $M$ et al (2015) Diffusionweighted magnetic resonance imaging for the prediction of pathologic response to neoadjuvant chemoradiotherapy in esophageal cancer. Radiother Oncol 115:163-170. https://doi.org/ 10.1016/j.radonc.2015.04.027

15. Fang P, Musall BC, Son JB et al (2018) Multimodal imaging of pathologic response to chemoradiation in esophageal cancer. Int $\mathrm{J}$ Radiat Oncol Biol Phys 102:996-1001. https://doi.org/10.1016/j. ijrobp.2018.02.029

16. Jacobs L, Intven M, van Lelyveld $\mathrm{N}$ et al (2016) Diffusionweighted MRI for early prediction of treatment response on preoperative chemoradiotherapy for patients with locally advanced rectal cancer. Ann Surg 263:522-528. https://doi.org/10.1097/SLA. 0000000000001311

17. Lambrecht M, Vandecaveye V, De Keyzer F et al (2012) Value of diffusion-weighted magnetic resonance imaging for prediction and early assessment of response to neoadjuvant radiochemotherapy in rectal cancer: preliminary results. Int J Radiat Oncol Biol Phys 82: 863-870. https://doi.org/10.1016/j.ijrobp.2010.12.063

18. De Cobelli F, Giganti F, Orsenigo E et al (2013) Apparent diffusion coefficient modifications in assessing gastro-oesophageal cancer response to neoadjuvant treatment: comparison with tumour regression grade at histology. Eur Radiol 23:2165-2174. https://doi.org/ 10.1007/s00330-013-2807-0

19. Harry VN (2010) Novel imaging techniques as response biomarkers in cervical cancer. Gynecol Oncol 116:253-261. https:// doi.org/10.1016/j.ygyno.2009.11.003

20. Partridge SC, Zhang Z, Newitt DC et al (2018) Diffusion-weighted MRI findings predict pathologic response in neoadjuvant treatment of breast cancer: the ACRIN 6698 Multicenter Trial. Radiology 289:618-627. https://doi.org/10.1148/radiol.2018180273

21. Padhani AR, Liu G, Koh DM et al (2009) Diffusion-weighted magnetic resonance imaging as a cancer biomarker: consensus and recommendations. Neoplasia 11:102-125

22. Thoeny HC, Ross BD (2010) Predicting and monitoring cancer treatment response with diffusion-weighted MRI. J Magn Reson Imaging 32:2-16. https://doi.org/10.1002/jmri.22167

23. Galbán CJ, Hoff BA, Chenevert TL, Ross BD (2017) Diffusion MRI in early cancer therapeutic response assessment. NMR Biomed 30

24. Wang L, Liu L, Han C et al (2016) The diffusion-weighted magnetic resonance imaging (DWI) predicts the early response of esophageal squamous cell carcinoma to concurrent chemoradiotherapy. Radiother Oncol 121:246-251. https://doi.org/10.1016/J. RADONC.2016.10.021

25. Wang L, Han C, Zhu S et al (2014) Investigation of using diffusionweighted magnetic resonance imaging to evaluate the therapeutic effect of esophageal carcinoma treatment. Oncol Res Treat 37:112-116. https://doi.org/10.1159/000360210

26. Giganti F, Salerno A, Ambrosi A et al (2016) Prognostic utility of diffusion-weighted MRI in oesophageal cancer: is apparent diffusion coefficient a potential marker of tumour aggressiveness? Radiol Med 121:173-180. https://doi.org/10.1007/s11547-0150585-2

27. Aoyagi T, Shuto K, Okazumi S et al (2011) Apparent diffusion coefficient values measured by diffusion-weighted imaging predict chemoradiotherapeutic effect for advanced esophageal cancer. Dig Surg 28:252-257. https://doi.org/10.1159/000328770

28. Heethuis SE, Borggreve AS, Goense L et al (2018) Quantification of variations in intra-fraction motion of esophageal tumors over the course of neoadjuvant chemoradiotherapy based on cine-MRI. Phys Med Biol 63. https://doi.org/10.1088/1361-6560/aacfb5
29. Boekhoff M, Kotte A, Mook S et al (2018) PO-0951: what CTV-toPTV margins are required for esophageal cancer radiotherapy? Radiother Oncol 127:S519-S520. https://doi.org/10.1016/S01678140(18)31261-1

30. Sobin L, Gospodarowicz M, Wittekind C (2009) TNM classification of malignant tumours, 7th edn. Wiley-Liss, New York

31. Mandard AM, Dalibard F, Mandard JC et al (1994) Pathologic assessment of tumor regression after preoperative chemoradiotherapy of esophageal carcinoma. Clinicopathologic correlations. Cancer 73:2680-2686

32. Musall B (2017) Quantitative DWI as an early imaging biomarker of the response to chemoradiation in esophageal cancer. UT GSBS dissertations and theses (Open Access). Available via: https:// digitalcommons.library.tmc.edu/utgsbs_dissertations $/ 805$

33. Yushkevich PA, Piven J, Hazlett HC et al (2006) User-guided 3D active contour segmentation of anatomical structures: significantly improved efficiency and reliability. Neuroimage 31:1116-1128. https://doi.org/10.1016/J.NEUROIMAGE.2006.01.015

34. Bol GH, Kotte ANTJ, van der Heide UA, Lagendijk JJW (2009) Simultaneous multi-modality ROI delineation in clinical practice. Comput Methods Programs Biomed 96:133-140. https://doi.org/ 10.1016/j.cmpb.2009.04.008

35. Van Buuren S, Groothuis-Oudshoorn K (2011) Multivariate imputation by chained equations. J Stat Softw 45:1-67. https://doi.org/ 10.18637/jss.v045.i03

36. Friedman J, Hastie T, Tibshirani R (2010) Regularization paths for generalized linear models via coordinate descent. J Stat Softw 33:122

37. Frank E Harrell Jr with contributions from Charles Dupont and many others (2018) Hmisc: Harrell miscellaneous. R package version 4.1-1

38. Perneger TV (1998) What's wrong with Bonferroni adjustments. BMJ 316:1236-1238. https://doi.org/10.1136/BMJ.316.7139.1236

39. Goense L, Borggreve AS, Heethuis SE et al (2018) Patient perspectives on repeated MRI and PET/CT examinations during neoadjuvant treatment of oesophageal cancer. Br J Radiol 91:20170710. https://doi.org/10.1259/bjr.20170710

40. Cheng B, Yu J (2018) Predictive value of diffusion-weighted MR imaging in early response to chemoradiotherapy of esophageal cancer: a meta-analysis. Dis Esophagus:1-6. https://doi.org/10.1093/ dote/doy065

41. Imanishi S, Shuto K, Aoyagi T et al (2013) Diffusion-weighted magnetic resonance imaging for predicting and detecting the early response to chemoradiotherapy of advanced esophageal squamous cell carcinoma. Dig Surg 30:240-248. https://doi.org/10.1159/ 000351435

42. Li Q-W, Qiu B, Wang B et al (2018) Prediction of pathologic responders to neoadjuvant chemoradiotherapy by diffusion-weighted magnetic resonance imaging in locally advanced esophageal squamous cell carcinoma: a prospective study. Dis Esophagus 31

43. Kwee RM, Dik AK, Sosef MN et al (2014) Interobserver reproducibility of diffusion-weighted MRI in monitoring tumor response to neoadjuvant therapy in esophageal cancer. PLoS One 9:e92211. https://doi.org/10.1371/journal.pone.0092211

44. Eisenhauer EA, Therasse P, Bogaerts J et al (2009) New response evaluation criteria in solid tumours: revised RECIST guideline (version 1.1). Eur J Cancer 45:228-247. https://doi.org/10.1016/j.ejca. 2008.10.026

45. Ilett E, Langer S, Olsen I et al (2015) Neuroendocrine carcinomas of the gastroenteropancreatic system: a comprehensive review. Diagnostics (Basel) 5:119-176. https://doi.org/10.3390/ diagnostics5020119

46. van der Veen A, Seesing MFJ, Wijnhoven BPL et al (2018) Management of resectable esophageal and gastric (mixed adeno) neuroendocrine carcinoma: a nationwide cohort study. Eur J Surg Oncol 44:1955-1962. https://doi.org/10.1016/J.EJSO.2018.07.058 
47. Lever FM, Lips IM, Crijns SPM et al (2014) Quantification of esophageal tumor motion on cine-magnetic resonance imaging. Int J Radiat Oncol Biol Phys 88:419-424. https://doi.org/10.1016/ j.ijrobp.2013.10.036

48. Heethuis SE, van Rossum PSN, Lips IM et al (2016) Dynamic contrast-enhanced MRI for treatment response assessment in patients with oesophageal cancer receiving neoadjuvant chemoradiotherapy. Radiother Oncol 120:128-135. https://doi.org/10.1016/j. radonc.2016.05.009

49. Goense L, Heethuis SE, van Rossum PSN et al (2018) Correlation between functional imaging markers derived from diffusionweighted MRI and 18F-FDG PET/CT in esophageal cancer. Nucl Med Commun 39:60-67. https://doi.org/10.1097/MNM. 0000000000000771

50. Heethuis SE, Goense L, van Rossum PSN et al (2018) DW-MRI and DCE-MRI are of complementary value in predicting pathologic response to neoadjuvant chemoradiotherapy for esophageal cancer. Acta Oncol 57:1201-1208. https://doi.org/10.1080/0284186X. 2018.1473637
51. Findlay JM, Dickson E, Fiorani C, et al (2019) Temporal validation of metabolic nodal response of esophageal cancer to neoadjuvant chemotherapy as an independent predictor of unresectable disease, survival, and recurrence. Eur Radiol https://doi.org/10.1007/ s00330-019-06310-9

52. Jin X, Zheng X, Chen D et al (2019) Prediction of response after chemoradiation for esophageal cancer using a combination of dosimetry and CT radiomics. Eur Radiol 29:6080-6088. https://doi. org/10.1007/s00330-019-06193-w

53. Borggreve AS, Mook S, Verheij M et al (2018) Preoperative imageguided identification of response to neoadjuvant chemoradiotherapy in esophageal cancer (PRIDE): a multicenter observational study. BMC Cancer 18:1006. https://doi.org/10.1186/s12885-018$4892-6$

Publisher's note Springer Nature remains neutral with regard to jurisdictional claims in published maps and institutional affiliations. 